\title{
Arbeitsmarktpolitik im Umbruch - Eine Chance für ältere Arbeitnehmer?
}

Ältere Arbeitnehmer rücken zunehmend in den Fokus der Politik. Insbesondere die von der Bundesregierung ins Leben gerufene Initiative 50plus soll durch arbeitsmarktpolitische Maßnahmen und das Werben für eine neue „altenfreundliche Unternehmenskultur" die Beschäftigungschancen Älterer nachhaltig verbessern. Vorruhestandsregelungen und Kündigungsschutz stehen im selben Atemzug zur Disposition. Im Entwurf für ein Gesetz zur Verbesserung der Beschäftigungschancen älterer Menschen setzt die Politik einseitig auf Instrumente, die die Einstellung älterer Arbeitnehmer finanziell lohnenswert erscheinen lassen. Wie effektiv ist die eingeschlagene Politikstrategie? Welche Konsequenzen hat sie für Ältere?

\section{Die Beschäftigungssitua- tion älterer Menschen}

Die Arbeitsmarktsituation älterer Arbeitnehmer ist im Vergleich zu jüngeren Erwerbspersonen nach wie vor schlecht. Sie ist gekennzeichnet durch ein unterdurchschnittliches Beschäftigungsniveau, hohe und verfestigte Arbeitslosigkeit sowie geringe Reintegrationschancen. Die Beschäftigungsquote älterer Arbeitnehmer (55 bis 64 Jahre) liegt mit knapp $46 \%$ (2006) nach wie vor deutlich unter derjenigen jüngerer Erwerbstätiger. Knapp $77 \%$ aller 25-bis 45 Jährigen zählten zum selben Zeitpunkt zu den Erwerbstätigen. Die Beschäftigungsquoten der Männer sind dabei in beiden Altersgruppen deutlich höher als die der Frauen, und qualifizierte ältere Arbeitnehmer haben im Vergleich zu unqualifizierten Personen eine höhere Chance, beschäftigt $\mathrm{zu}$ sein und es auch zu bleiben (Bosch/ Schief 2005). Spiegelbildlich dazu ist die hohe Arbeitslosigkeit Älterer zu lesen: Im Dezember 2006 betrug die Arbeitslosenquote der über 50-Jährigen 26,6\%; bei den über 55-Jährigen lag sie bei 13,0 \% (BA 2006a). Ältere Arbeitslose sind dabei überdurchschnittlich lange arbeitslos. Arbeitslose, die im Jahr 2006 eine Beschäftigung aufnahmen, waren zuvor im Schnitt 36 Wochen ohne Job, während die über 50Jährigen durchschnittlich erst nach 52 Wochen eine neue Stelle fanden (Dietz et al. 2006). Nur $23 \%$ aller älteren Arbeitnehmer ab 55 Jahren beendeten ihre Arbeitslosigkeit durch die Aufnahme einer Erwerbstätigkeit (einschließlich geförderter Be- schäftigung). Die Hälfte der erfolgten Abgänge aus Arbeitslosigkeit waren Übergänge in die Nichterwerbstätigkeit (Engstler/ Brussig 2006).

Die geringe Teilhabe Älterer am Erwerbsleben wird zunehmend kritisch diskutiert und eine höhere Integration Älterer in den Arbeitsmarkt für notwendig gehalten. Die Bundesregierung hat dementsprechend im Dezember 2006 einen Entwurf für ein Gesetz zur Verbesserung der Beschäftigungschancen älterer Menschen (Verbesserungsgesetz) ins Gesetzgebungsverfahren eingebracht. Es ist Bestandteil der Initiative 50plus, mit der nicht nur ein arbeitsmarktpolitischer Strategiewechsel hin zu mehr Erwerbstätigkeit Älterer angestrebt wird, sondern auch für ein betriebliches Personalwesen geworben wird, das auf das Älterwerden der Beschäftigten reagiert. Diesem verstärkten Engagement der Politik liegen im Wesentlichen drei Motive zugrunde: Bedingt durch den Rückgang der Bevölkerung in der Altersgruppe der 20bis 59-Jährigen und den gleichzeitigen Anstieg in der Altersgruppe der über 60-Jährigen scheint erstens eine nachhaltige Finanzierung der sozialen Sicherungssysteme nur dann möglich, wenn die Beschäftigungs- und damit auch die Beitragsdauer aller Beschäftigten ausgedehnt wird (Promberger/Wübbeke 2006). Zweitens wird die Erschließung der großen ungenutzten Personalreserven bei Älteren als notwendig erachtet, um auch bei einer veränderten altersstrukturellen Zusammensetzung die ökonomische Innovationsfähigkeit der Gesellschaft zu erhalten. Drittens ist eine größere Erwerbsbeteiligung Älterer auch ein Beitrag zur Vermeidung von Altersarmut, da ein langer Sozialleistungsbezug vor dem Renteneintritt das Rentenniveau nachhaltig senkt.

Bereits mit dem ersten Hartz-Gesetz wurden im Jahr 2003 Instrumente eingeführt, die die Beschäftigungsquote Älterer erhöhen sollten und die nun durch das Verbesserungsgesetz ausgebaut werden sollen. Vor diesem Hintergrund untersucht dieser Beitrag sowohl die Effektivität des Instrumentariums als auch die Konsequenzen für Betroffene. Zunächst stellen wir kurz die Grundzüge der Arbeitsmarktpolitik für Ältere seit der Hartz-Reform dar. In Abschnitt 3 diskutieren wir den eingeschlagenen Reformweg vor dem Hintergrund jüngster Evaluierungsergebnisse. In Abschnitt 4 beschreiben wir prekäre Altersübergänge als eine wesentliche Konsequenz der aktuellen Arbeitsmarktpolitik für Ältere. Im fünften Teil gehen wir der Frage nach, ob eine weitergehende Flexibilisierung des Arbeitsrechts ein Weg sein kann, um die Beschäftigungschancen Älterer zu erhöhen. Im Schlussteil weisen wir auf Reformperspek-

Stefanie Kremer ist Wissenschaftlerin im WSI in der Hans-Böckler-Stiftung. Arbeitsschwerpunkt: Projekt Regulierung des Arbeitsmarktes (REGAM);

e-mail: stefanie-kremer@boeckler.de Judith Aust, Dr., ist Wissenschaftlerin im WSI in der Hans-Böckler-Stiftung. Arbeitsschwerpunkte: Arbeitsmarktpolitik, vergleichende Wohlfahrtsstaatsforschung. e-mail: judith-aust@boeckler.de

Die Autorinnen danken Silke Bothfeld und Nadine Zeibig für wertvolle Hinweise. 
tiven hin, die unserer Einschätzung zufolge besser geeignet wären, mit der problematischen Situation Älterer auf dem Arbeitsmarkt umzugehen.

\section{Arbeitsmarktpolitik für Ältere?}

Die zwischen 2003 und 2005 verabschiedeten Strukturreformen zielten darauf, durch ein neues Arrangement von Anreizen und Sanktionen die Lage am Arbeitsmarkt nachhaltig zu verbessern. Mehr Eigenverantwortung der Arbeitsuchenden war dabei die Schlüsselbotschaft (Seifert 2006). Der Idee einer stärkeren Eigenverantwortung entspricht ein Leistungsrecht, das sich an den Prinzipien des Förderns und Forderns orientiert. Was aber zunächst nach einem ausbalancierten Nebeneinander zweier gleichberechtigter Handlungsmaximen klingt, entpuppt sich bei genauerer Analyse als eine umfangreiche Liste an sozialen Einschnitten, sanktionierenden Regularien und arbeitsrechtlichen Flexibilisierungsbestrebungen.

Ältere Arbeitnehmer sind von diesem politischen Strategiewechsel nicht ausgenommen. Dennoch liegen den für diese Gruppe zusätzlich verabschiedeten Maßnahmen eine zielgruppenspezifische Problemwahrnehmung und ein spezieller, politikfeldübergreifender Reformansatz zugrunde. Es wird davon ausgegangen, dass die vielfältigen Möglichkeiten eines vorzeitigen Ausstiegs aus dem Erwerbsleben die geringe Erwerbsbeteiligung Älterer befördern und es Unternehmen erleichtern, im Zuge von Restrukturierungsmaßnahmen Ältere „sozialverträglich“ zu entlassen (Eichhorst/Sproß 2005). Ein weiteres Argument verweist auf die verhältnismäßig hohen Arbeitslosengeldbezüge Älterer, die aus langen Beitragszahlungen resultieren. Sie würden den Anreiz zur zügigen Wiederaufnahme einer Beschäftigung verringern (Dietz et al. 2006). Schließlich wird angenommen, dass die Kündigungsschutzregelungen die Einstellungsbereitschaft bei Arbeitgebern reduzieren und damit eine Segmentierung des Arbeitsmarktes stützen. Ein umfassender Kündigungsschutz stärke diejenigen, deren Arbeitsmarktposition von vornherein relativ stark ist (Sesselmeier 2004, S. 127). Die Beschäftigungschancen der am Arbeitsmarkt be- nachteiligten Gruppen, wie z. B. Frauen und Ältere, nähmen weiter ab. Durch eine Verringerung des Kündigungsschutzes könne eine größere Dynamik auf dem Arbeitsmarkt erreicht werden, die die Beschäftigungschancen der jetzt Benachteiligten vergrößere (Sachverständigenrat 2006, S. 412ff.).

An dieser Problemwahrnehmung orientiert sich die aktuelle arbeitsmarktpolitische Strategie für Ältere. Zahlreich sind die Einschränkungen im Rentenrecht: Das geplante Auslaufen der Altersteilzeitregelung, die Anhebung der Altersgrenze für den Bezug der Altersrenten für Frauen und für langjährig Versicherte auf 65 Jahre; des Weiteren die Anhebung der Altersgrenze für den Bezug der Altersrenten wegen Arbeitslosigkeit, nach Altersteilzeit und für Schwerbehinderte auf 63 Jahre und die Einführung von Abschlägen von bis zu 3,6 \% pro Jahr bei einer vorgezogenen Verrentung. Alle diese Maßnahmen minimieren die Möglichkeiten, vor dem Erreichen der derzeitigen Regelaltersgrenze in den Ruhestand zu gehen. Die Reduzierung der maximalen Dauer des Arbeitslosengeldbezugs für alle über 55-jährigen Arbeitslosen auf 18 Monate schränkt Transferleistungen ein und verkürzt die durch die Arbeitslosenversicherung finanzierten Warteschleifen auf den Vorruhestand. Auf diese Weise werden Folgekosten von Arbeitslosigkeit individualisiert. Als Folge der Zusammenlegung von Arbeitslosenhilfe und Sozialhilfe erhalten viele ältere ehemalige Arbeitslosenhilfeempfänger deutlich geringere oder im Fall einer Bedarfsgemeinschaft gegebenenfalls gar keine Leistungen mehr.

Diesen Leistungseinschränkungen steht ein vergleichsweise bescheidenes Repertoire an Förderinstrumenten gegenüber (Aust et al. 2006). Die Entgeltsicherung ( $\$ 421$ j SGB III), der Beitragsbonus ( $\$ 421 \mathrm{k}$ SGB III) und der Eingliederungszuschuss für Ältere ( $\$ \$ 218$, 421f SGB III) sind Maßnahmen, die die Rückkehr älterer Arbeitsloser durch spezielle Zuschüsse an Arbeitgeber und Arbeitnehmer fördern sollen. Dazu soll auch die als Flexibilisierung verstandene Senkung des Kündigungsschutzniveaus für Arbeitnehmer ab 52 Jahren beitragen. Die besondere Befristungsregelung für Ältere im $\$ 14$ Abs.3 TzBfG wurde erstmals 2001 mit einer Mindestaltersgrenze von 58 Jahren geschaffen; die Herabsenkung der Altersgrenze auf 52 Jahre erfolgte 2003. Im Jahr 2005 wurde sie für europarechtswidrig erklärt.

\section{Erfolglose Aktivierung und Sozialleistungsabbau}

Arbeitsmarktpolitik kann nicht alles. Der Abbau der hohen und sich verfestigenden Arbeitslosigkeit gerade bei „Problemgruppen“ ist prinzipiell nur durch eine gesamtwirtschaftliche Strategie der Koordinierung der Lohn-, Geld- und Finanzpolitik zu lösen. Besonders international vergleichende Studien zeigen, dass nicht allein die Ausgestaltung des Transfersystems und die spezifische Gewichtung der Grundprinzipien des Förderns und Forderns von Bedeutung sind. Ein weiterer Erfolgsfaktor, insbesondere bei Ländern mit einer positiven Beschäftigungsbilanz wie Dänemark oder Finnland, war der wirtschaftliche Aufschwung, der der Trendwende bei der Beschäftigung vorausging oder diese zumindest begleitete (Kraatz et al. 2006). Ebenso wichtig ist die Ausrichtung der betrieblichen Personalpolitik, bei der trotz zahlreicher Bemühungen (z. B. Initiative 50plus) der Strategiewechsel noch keine eindeutigen Erfolge mit sich gebracht hat. Nur die Hälfte aller Betriebe ist bereit - so die Ergebnisse des IAB-Betriebspanels - ältere Bewerber ohne finanzielle Zuschüsse einzustellen, etwa ein Drittel knüpft eine mögliche Einstellung an Bedingungen wie Lohnkostenzuschüsse, und jeder siebte Betrieb lehnt die Einstellung Älterer grundsätzlich ab. Darüber hinaus konnte gezeigt werden, dass die jeweils letzte Neueinstellung in nur ca. 10 \% der Fälle eine Person im Alter von 50 Jahren oder älter war (Bellmann/Leber 2005).

Dennoch kann Arbeitsmarktpolitik die Feinmechanismen in den Anpassungsprozessen am Arbeitsmarkt gestalten (Bothfeld 2005, S. 419). Ihre Aufgabe ist es, das Ungleichgewicht zwischen Angebot und Nachfrage durch die Arbeitsvermittlung und -beratung zu verbessern, Eingliederungschancen für Personen zu erhöhen, die geringe Aussichten haben, eigenständig wieder eine Beschäftigung zu finden, und die Dauer der Arbeitslosigkeit durch die Beschleunigung von Suchprozessen zu verkürzen. Ausreichende Lohnersatzleistungen in Phasen der Arbeitslosigkeit sind eine Grundlage nicht nur zur Verhinderung von Armut, sondern sie haben auch eine sozialinvestive Komponente: Sie schaffen Handlungsspielräume bei der Suche nach 
einer angemessenen Beschäftigung und tragen zum Erhalt von Qualifikationen bei (Gangl/Schmidt 2002).

\subsection{WAS SAGT DIE EVALUIERUNGSFORSCHUNG?}

Die Instrumente zur Unterstützung der Reintegration Älterer in den Arbeitsmarkt sollen durch das geplante Gesetz zur Verbesserung der Beschäftigungschancen älterer Menschen weiter ausgebaut werden. Dazu gehören der spezielle Eingliederungszuschuss und die Entgeltsicherung für Arbeitnehmer ab 50 Jahren sowie der Beitragsbonus für Arbeitnehmer ab 55 Jahren. Die beschäftigungspolitische Bilanz der jüngsten Evaluierungsergebnisse fällt allerdings ernüchternd aus. Laut den Erkenntnissen des Bundesministeriums für Arbeit und Soziales zur Wirksamkeit moderner Dienstleistungen am Arbeitsmarkt hatten weder der Beitragsbonus noch die Entgeltsicherung noch der Eingliederungszuschuss Einfluss auf die Beschäftigungschancen Älterer (BMAS 2006a, S. 87).

Der Beitragsbonus befreit den Arbeitgeber von seinen Beiträgen zur Arbeitslosenversicherung und den Arbeitnehmer selbst zur Hälfte. Diese Regelung ist bisher nur für Arbeitsverhältnisse vorgesehen, die vor dem 1.1.2008 abgeschlossen werden. Während im Jahr 2003 noch rund 9.000 neue Beschäftigungsverhältnisse zustande gekommen sind, für die Arbeitgeber den Beitragsbonus in Anspruch nahmen, waren es im Jahr 2004 nur noch rund 3.600 (ebd., S. 187). Es konnte kein Einfluss des Instruments auf die Beschäftigungschancen festgestellt werden. Diese Einschätzung deckt sich auch mit Aussagen von 30 geförderten Betrieben, die in der Mehrheit die entsprechenden Personen auch ohne Beitragsbonus eingestellt hätten (ebd., S. 188).

Ähnlich erfolglos ist das Instrument der Entgeltsicherung (ebd., S. 192). Die Entgeltsicherung ist ein befristetes KombilohnModell, bei dem der Arbeitnehmer einen Lohnzuschuss und die Erstattung eines Teils seines Rentenversicherungsbeitrags erhält. Er muss einen Restanspruch auf Arbeitslosengeld von mindestens 180 Tagen haben, und der neue - tarifliche oder übliche Lohn muss mindestens $50 €$ niedriger sein als der vorherige. Für die Dauer des verbleibenden Arbeitslosengeldanspruchs werden $50 \%$ der Differenz zwischen dem alten und dem neuen Lohn ausgeglichen. Die erforderliche Mindestdauer des Arbeitslosen- geldanspruchs soll laut dem Reformvorschlag um 60 Tage gekürzt werden, wodurch die Anspruchsvoraussetzungen erleichtert würden. Zudem soll die Höchstbezugsdauer einheitlich zwei Jahre betragen, wobei im zweiten Jahr nur noch $30 \%$ der Differenz ausgeglichen werden sollen. Im Jahr 2005 gab es rund 5.300 Zugänge in die Entgeltsicherung (ebd., S. 188). Die Mehrheit der Geförderten erhielt einen Arbeitsentgeltzuschuss von unter $285 €$ monatlich. Ein Grund für die geringe Inanspruchnahme war, dass Arbeitsvermittler eher mit Instrumenten arbeiten, die die Einstellungsbereitschaft beim Arbeitgeber erhöhen (Dietz et al. 2006, S. 4). Ergebnisse von Telefonbefragungen verweisen zudem darauf, dass spürbare Mitnahmeeffekte zu beobachten waren: Fast ein Drittel der Befragten gab an, dass sie die Beschäftigung auch ohne Entgeltsicherung angenommen hätten. $65 \%$ sagten, dass die Entgeltsicherung die Beschäftigungsaufnahme zwar erleichtert, aber nicht ausgelöst habe. Nur $8 \%$ gaben an, dass sie ohne die Entgeltsicherung die neue Stelle nicht angenommen hätten (BMAS 2006a, S. 192).

Den Eingliederungszuschuss für Ältere erhält ein Arbeitgeber, der einen älteren Arbeitnehmer mit Vermittlungshemmnissen einstellt. Künftig soll allerdings statt eines Vermittlungshemmnisses bereits ein festgestellter individueller Unterstützungsbedarf die Förderungswürdigkeit begründen, wenn der Arbeitnehmer zudem mindestens sechs Monate arbeitslos war. Der maximal 36 Monate gewährte Zuschuss beträgt 30 bis $50 \%$ des Arbeitsentgelts und verringert sich jährlich um $10 \%$. Im Zeitraum Januar bis August 2006 lag die Zahl der Förderzugänge bei rund 18.500 (BA 2006b). Ein Grund für die geringe Inanspruchnahme und Wirkungsweise dieses Instruments ist, dass der Eingliederungszuschuss ähnlich wie die beiden anderen Instrumente ein nur nachgelagertes Entscheidungskriterium für die Einstellung eines Bewerbers ist. Aber auch schlechte Beziehungen zu den Agenturen für Arbeit spielen eine Rolle.

\subsection{LEISTUNGSKÜRZUNGEN}

Von den Einschränkungen der finanziellen Leistungen im Zuge der Hartz-Reform sind ältere Erwerbslose besonders betroffen. Engstler/Brussig (2006) kommen in einer Studie zur Arbeitslosigkeit am Ende des Erwerbslebens zu folgendem Ergebnis: Im Au- gust 2006 bezogen $45,4 \%$ der arbeitslos Registrierten ab 50 Jahren Arbeitslosengeld. Von allen Arbeitslosen gehörten nur 35,6 \% zum Rechtskreis des SGB III. Ältere Arbeitslose erhielten damit überdurchschnittlich oft Leistungen aus dem SGB III. Dies könnte sich durch die Verkürzung der maximalen Bezugsdauer des Arbeitslosengeldes ebenso wie durch die schrittweise Heraufsetzung des frühestmöglichen Bezugs einer Altersrente bald ändern. Denn bereits 2005 erfolgten $30 \%$ der Abgänge aus dem Arbeitslosengeld wegen Erschöpfung des Leistungsbezugs (ebd., S. 4). Es ist dabei davon auszugehen, dass der Übergang in die neue Grundsicherung für arbeitslose Ältere mit besonders hohen finanziellen Verlusten verbunden ist. Ältere Arbeitslose hatten im Vergleich zu jüngeren Arbeitslosen aufgrund höherer Einkommen in ihrer letzten Beschäftigung höhere Ansprüche an die Arbeitslosenhilfe und sind dementsprechend stärker von den Leistungseinschränkungen durch die Zusammenlegung von Arbeitslosenhilfe und Sozialhilfe zur Grundsicherung für Arbeitslose betroffen. Sie hatten zudem mehr Gelegenheit, Ersparnisse zu bilden und sind häufiger verheiratet, sodass Einkommens- und Vermögensanrechnungen wahrscheinlicher sind (Blos/Rudolph 2005). Als besonders problematisch erweist sich in diesem Kontext auch die sogenannte 58er-Regelung ( $\$ 428$ SGB III), die zunächst nur bis Ende 2007 gilt. Trotz eines weitergehenden Leistungsbezugs müssen ältere Arbeitnehmer hiernach dem Arbeitsmarkt nicht mehr zur Verfügung stehen. Gleichzeitig sind sie verpflichtet, zum frühestmöglichen Zeitpunkt eine abschlagsfreie Rente zu beantragen. Da dieser Zeitpunkt in den letzten Jahren deutlich nach hinten verschoben wurde, ${ }^{1}$ müssen und werden sich zunehmend mehr ältere Arbeitslose im Status des Leistungsbezugs mit Abschlägen befinden. Die Zahl der Personen, die von der 58er-Regelung Gebrauch gemacht haben, ist bereits von $204.000 \mathrm{im}$ Jahr 2000 auf fast 400.000 im Jahr 2004 gestiegen (OECD 2005, S. 89). $75 \%$ der Arbeitslosen über 58 Jahren nahmen 2003 diese Regelung in Anspruch. Eine Auswertung der Arbeitslosigkeit Älterer speziell im Hin-

\footnotetext{
Dies gilt sowohl für die besonderen Rentenarten als auch für die Regelaltersrente. Im März 2007 wurde die Heraufsetzung des gesetzlichen Rentenalters auf 67 Jahre im Bundestag beschlossen. Die schrittweise Anhebung wird zwischen 2012 und 2029 erfolgen.
} 
blick auf die 58er-Regelung führt sogar zu der Feststellung, dass Ältere besonders zur Inanspruchnahme dieser Regelung gedrängt wurden, seitdem Ende der 1990er Jahre der Wechsel zur aktivierenden Arbeitsmarktpolitik vollzogen wurde und die Möglichkeiten, in Vorruhestand zu gehen, eigentlich verringert werden sollten (Brussig/Knuth 2006, S. 310). Die damit verbundenen langfristigen finanziellen Konsequenzen sind erheblich: Arbeitslosengeld II-Empfänger erwerben kaum zusätzliche Rentenansprüche. Ein Jahr Arbeitslosengeld II-Bezug führt zu einem zusätzlichen monatlichen Rentenanspruch von 2,18 € (ebd., S. 307). Personen, die aufgrund eines Partnereinkommens oder eigenen Vermögens kein Arbeitslosengeld II bekommen, sind gezwungen, ihre vorhandenen finanziellen Ressourcen aufzulösen, ohne weitere Rentenansprüche zu erwerben.

\section{$\angle$ \\ Die Konsequenz: Prekäre Altersübergänge}

Vor dem Hintergrund der hohen Arbeitslosigkeit Älterer und der gleichzeitigen Heraufsetzung der Altersgrenze für die Regelaltersrente auf 67 Jahre ist davon auszugehen, dass zunehmend mehr Menschen über einen länger werdenden Zeitraum von Leistungen der Arbeitslosenversicherung bzw. Grundsicherung oder ganz ohne staatliche Unterstützung und Erwerbseinkommen leben müssen. Dies bestätigen auch die Ergebnisse des Altersübergangsmonitors, der sowohl den Renteneintritt als auch den Austritt aus der Erwerbstätigkeit analysiert.

Das durchschnittliche Rentenzugangsalter ist zwischen 1996 und 2005 um ein Jahr auf nun 63,1 Jahre gestiegen (Knuth et al. 2006). Der Anstieg ist dabei sowohl dem Motiv geschuldet, Abschläge zu vermeiden, als auch der Tatsache, dass viele Versicherte mit Vollendung des 62. Lebensjahrs die Zugangsvoraussetzungen für einen vorzeitigen Rentenbezug noch nicht erfüllt haben (ebd., S. 309). Bezogen auf den Geburtsjahrgang 1939 wird deutlich, dass es sich bei den Versicherten, die mit 65 Jahren in Rente gehen, überwiegend um Personen handelt, die wegen fehlender versicherungsrechtlicher Voraussetzungen keinen Zugang $\mathrm{zu}$ einer vorzeitigen Rente haben. Dies betrifft vor allem Frauen mit kurzen Versicherungszeiten (aufgrund geringer Zahl von
Erwerbsjahren) oder nicht erwerbstätige Frauen, die nur durch die Anrechnung von Kindererziehungszeiten einen eigenständigen Rentenanspruch erworben haben (Bäcker 2006). Trotz des steigenden Durchschnittsalters gehen jedoch - erkennbar am Rentenzugangsquotienten - anteilig mehr Menschen mit 60 Jahren in Rente. Auch wenn die Auswirkungen der Hartz-Reformen auf den Altersübergang statistisch noch nicht nachgewiesen werden können, spricht einiges dafür, dass die wieder steigende Inanspruchnahme des vorzeitigen Rentenbezugs trotz hoher Abschläge als Reaktion auf die strengen Bedarfsprüfungen für das Arbeitslosengeld II gewertet werden kann. Es ist daher von einer Polarisierung des Rentenzugangsalters und einer Zunahme der sozialen Differenzierung im Alter auszugehen.

Zudem ist der Zugang zur Altersrente für die ganz überwiegende Zahl der Älteren nicht mit einem Ausscheiden aus einer sozialversicherungspflichtigen Beschäftigung verbunden. Im Jahr 2004 waren vier Fünftel der Neurentner zuvor arbeitslos, in der Altersteilzeit oder nicht erwerbstätig (Bäcker 2006, S. 10). Insbesondere bei Männern führt Arbeitslosigkeit vor Rentenbeginn in über $70 \%$ der Fälle zu einem um die jeweiligen Abschläge verminderten Rentenzugang. Über die Hälfte der langzeitarbeitslosen Männer akzeptiert beim Rentenzugang sogar Abschläge von $18 \%$. Ein ähnliches Muster ist auch bei Frauen zu beobachten. Insgesamt ist davon auszugehen, dass sich die Kombination von längerer Arbeitslosigkeit und Abschlägen bei vorzeitigem Rentenbeginn negativ auf das Alterseinkommen auswirkt. Betroffen werden davon all jene sein, die aus gesundheitlichen oder qualifikatorischen Gründen dem Arbeitsmarkt nicht zur Verfügung stehen oder nicht mehr nachgefragt werden. Gleiches gilt für all die Personen, die aufgrund von ungenügender Versicherungszeit und zu erwartender niedriger Rentenhöhe im Arbeitslosengeld II-Bezug verbleiben müssen.

\section{Hilft Älteren mehr arbeitsrechtliche Flexibilität?}

Von Flexibilisierungs-Befürwortern werden gelockerte Kündigungsschutzregelun- gen als Möglichkeit gesehen, die Beschäftigungschancen arbeitslos gewordener Älterer zu erhöhen und eine zügige Reintegration in den Arbeitsmarkt zu stützen. Unseres Erachtens ist der Weg über einen gelockerten Kündigungsschutz jedoch nicht gangbar.

\subsection{PROBLEME DER BEFRISTUNGS- REGELUNG FÜR ÄLTERE}

Die Befristungsregelung ( $\$ 14$ Abs.3 TzBfG) soll älteren Arbeitnehmern zugute kommen, indem sie erlaubt, einen über 52Jährigen ohne sachlichen Grund, ohne zeitliche Höchstbegrenzung und beliebig oft hintereinander befristet einzustellen. In ihrer Fassung von 2001 galt die Vorschrift für alle über 58-Jährigen und wurde 2003 auf alle über 52-Jährigen ausgeweitet. Im November 2005 erklärte der Europäische Gerichtshof (EuGH) die Vorschrift jedoch für unzulässig, weil sie gegen die Antidiskriminierungsrichtlinie (2000/78/EG) und den allgemeinen Gleichheitsgrundsatz (EuGH 2005) ${ }^{2}$ verstoßen würde. Als Teil des Gesetzentwurfs zur Verbesserung der Beschäftigungschancen älterer Menschen liegt nun jedoch eine Neufassung der Vorschrift vor. Mit ihr wurde auf das Urteil des EuGH reagiert.

Bei der Beurteilung des EuGH standen die beschäftigungsfördernden Aspekte nicht im Mittelpunkt, vielmehr wurde die Regelung an den Maßgaben der Antidiskriminierungsrichtlinie und des allgemeinen Gleichheitsgrundsatzes gemessen. Der EuGH hat zwar eine beschäftigungspolitische Wirksamkeit der Vorschrift nicht ausgeschlossen, er hat sie jedoch auch nicht bestätigt. Stattdessen hielt er eine Unterscheidung zwischen älteren Arbeitnehmern, die aufgrund persönlicher Umstände eingeschränkte Arbeitsmarktchancen haben, und älteren Arbeitnehmern, die keine verminderten Arbeitsmarktchancen haben, für erforderlich. Lediglich für die erste Gruppe könnten Kündigungserleichterungen gerechtfertigt sein. Diese seien nur zulässig, wenn sie nicht an das Alter, sondern an die Integrationsbedürftigkeit eines Arbeitslosen anknüpfen. Der EuGH verwies auch auf die Gefahr, dass ältere Arbeitnehmer durch eine solche Regelung

\footnotetext{
2 Der Bundesgerichtshof hat die Entscheidung des EuGH in seinem Urteil vom 26.4.2006 (Az. 7 AZR 500/04) bestätigt.
} 


\begin{tabular}{|c|c|c|c|c|c|c|}
\hline & \multicolumn{5}{|c|}{ Lebensalter der Arbeitnehmer } & \multirow[t]{2}{*}{ Durchschnitt } \\
\hline & $\begin{array}{l}14-24 \\
\text { Jahre }\end{array}$ & $\begin{array}{l}25-34 \\
\text { Jahre }\end{array}$ & $\begin{array}{l}35-44 \\
\text { Jahre }\end{array}$ & $\begin{array}{l}\text { 45-54 } \\
\text { Jahre }\end{array}$ & $\begin{array}{l}55-65 \\
\text { Jahre }\end{array}$ & \\
\hline Eigenkündigung & 38 & 49 & 43 & 25 & 13 & 39 \\
\hline $\begin{array}{l}\text { Kündigung durch } \\
\text { Arbeitgeber }\end{array}$ & 25 & 26 & 31 & 44 & 47 & 32 \\
\hline Aufhebungsvertrag & 8 & 8 & 10 & 11 & 20 & 10 \\
\hline $\begin{array}{l}\text { Auslauf einer Befristung/ } \\
\text { eines Ausbildungsvertrages }\end{array}$ & 29 & 17 & 16 & 20 & 20 & 19 \\
\hline Gesamt & 100 & 100 & 100 & 100 & 100 & 100 \\
\hline Quelle: WSI 2001, eigene Berechn & wich & ebnisse & & & & $\begin{array}{l}\text { Hans Böckler } \\
\text { Stiftung }\end{array}$ \\
\hline
\end{tabular}

grundsätzlich von unbefristeten Beschäftigungsverhältnissen ausgeschlossen werden könnten. Er stellte fest, dass die Gruppe älterer Arbeitnehmer „während eines erheblichen Teils ihres Berufslebens Gefahr (laufe), von festen Beschäftigungsverhältnissen ausgeschlossen zu sein, die doch (...) einen wichtigen Aspekt des Arbeitnehmerschutzes darstellen“. Damit ging er auf Dis$\operatorname{tanz} z u$ den Flexibilisierungs-Befürworten, die er zudem ausdrücklich aufforderte, zu belegen, in welchem Ausmaß eine Einschränkung des Kündigungsschutzes sinnvoll sei.

Die Europarechtmäßigkeit der geplanten Neuregelung des $\$ 14$ Abs.3 TzBfG im Verbesserungsgesetz, die im Wesentlichen Vorschläge des Ausschusses Arbeitsrecht des Deutschen Anwaltvereins (Stellungnahme Nr. 54/2005 v. Dez. 2005) und von Thüsing (2005) aufgreift, ist ebenfalls umstritten. Voraussetzung für eine sachgrundlose, beliebig häufige Befristung für über 52-Jährige soll zum einen eine vorangehende viermonatige Beschäftigungslosigkeit sein, die auch Zeiten ohne Arbeitslosmeldung umfasst. Zum anderen schlägt der Gesetzgeber vor, die Befristungsmöglichkeit auf fünf Jahre zu begrenzen. Im Extremfall wäre es zulässig, einen Arbeitnehmer fünf Jahre lang für jeweils einen Tag einzustellen. Diese Regelung bewegt sich laut Preis (2006, S. 410) an der Grenze des Zulässigen. Denn erneut wird hiermit zwar nicht nur, aber auch an das Alter angeknüpft. Soll mit Kündigungserleichterungen auf verminderte Beschäftigungschancen reagiert werden, darf aber nur am Sachproblem, nicht am Alter angesetzt werden. Ähnlich sieht es auch der DGB, dem zufolge die Kriterien so differenziert formuliert werden müssten, dass eine spezielle Kündigungserleichterung für Ältere sinnvollerweise entfallen sollte (DGB 2006).
Befürworter der Regelung nehmen an, dass eine erweiterte Befristungsmöglichkeit für Ältere die Einstellungsbereitschaft bei Arbeitgebern erhöht. Gegen diese Annahme spricht jedoch die seit der ersten Fassung der Vorschrift zu beobachtende Entwicklung bei den befristeten Verträgen mit Älteren. Deren Zahl hat sich seit 2000 nicht erhöht. $\mathrm{Zu}$ diesem Zeitpunkt waren $4,6 \%$ der 55 - bis 65 -jährigen versicherungspflichtig Beschäftigten befristet angestellt, fünf Jahre später betrug ihr Anteil 4,5\% (Mikrozensus 2000; 2005). Ein Grund für die geringe Inanspruchnahme der Befristungsmöglichkeit für Ältere könnten zwar die anhaltenden Diskussionen um die Vereinbarkeit mit dem Europarecht gewesen sein. Denn schon bei der erstmaligen Verabschiedung der Vorschrift wurden europarechtliche Bedenken laut. ${ }^{3}$ Sie hielten aber noch nicht einmal den Gesetzgeber davon ab, die Regelung 2003 durch Herabsetzen der Altersgrenze weiter zu verschärfen. Arbeitgeber wurden zudem mit der Einschätzung beruhigt, dass ein böses Erwachen ausgeschlossen sei (Wiedemann/Thüsing 2002). Wahrscheinlicher ist daher, dass sich hier die allgemeinen Bedenken gegen die beschäftigungspolitische Wirksamkeit von Kündigungserleichterungen bestätigt haben: Denn auch empirische Untersuchungen zu den Auswirkungen von Kündigungsschutzregelungen auf das gesamte Beschäftigungsniveau, die alle Altersgruppen und alle Arten von Kündigungserleichterungen umfassen, konnten bisher keine Wirkungen feststellen (OECD 2004).

\subsection{DER MYTHOS DER UNKÜNDBARKEIT}

Älteren Arbeitnehmern kann ebenso wie ihren jüngeren Kollegen gekündigt werden. Eine Analyse der Kündigungspraxis - auf
Basis einer WSI-Befragung zur Beendigung von Arbeitsverhältnissen im Jahr 2001 zeigt, dass älteren Arbeitnehmern (55 bis 65 Jahre) im Vergleich zu jüngeren Altersgruppen sogar deutlich häufiger gekündigt wird (Tabelle 1). Dagegen ist die Zahl der Eigenkündigungen bei älteren Arbeitnehmern mit $13 \%$ weit unterdurchschnittlich. Ältere halten demnach deutlich häufiger an ihrem Arbeitsplatz fest als Jüngere. Gleichzeitig verlieren sie ihren Arbeitsplatz jedoch öfter unfreiwillig. Mit $47 \%$ sind die Vertragsbeendigungen durch arbeitgeberseitige Kündigungen bei den Älteren weit überdurchschnittlich. Der Gesamtdurchschnitt aller Arbeitgeberkündigungen liegt bei $32 \%$. Arbeitgeber wissen offenbar, dass und wie sie Älteren kündigen können. Eine besondere Befristungsregelung für Ältere würde den Trend des unfreiwilligen Ausscheidens weiter verstärken.

Die gesetzlichen Vorschriften stellen keine besonderen Anforderungen an die Kündigung eines Älteren - entgegen einer weit verbreiteten Ansicht: Wer über 50 Jahre alt ist, könne kaum noch gekündigt werden, weil Gesetze das verhindern würden (Lotter 2006). Die OECD spricht ebenfalls von strengen Kündigungsschutzbestimmungen für Ältere, verweist hier jedoch nur auf eine altersunspezifische Studie zur Rigidität des Kündigungsschutzes (OECD 2005, S. 125). ${ }^{4}$ Fakt ist: Besondere Kündigungsschutzbestimmungen für ältere Arbeitnehmer bestehen nicht. Zwar ist das Le-

\footnotetext{
3 Diese Bedenken hatten sich größtenteils nicht auf die Antidiskriminierungsrichtlinie, sondern auf die Befristungsrichtlinie (1999/70/EG) gestützt, die im konkreten Fall nicht einschlägig war.

4 Die einzige Sonderregelung für Ältere, die im OECD-Bericht angesprochen wird, ist eine sozialrechtliche, die Erstattungspflicht des Arbeitgebers nach $\S 147$ a SGB III. Unter besonderen Voraussetzungen müssen Arbeitgeber im Fall der Entlassung eines langjährigen Mitarbeiters über 55 Jahre das an ihn gezahlte Arbeitslosengeld für längstens 32 Monate erstatten. Die Erstattungspflicht trifft jedoch nur einen von 100 Arbeitgebern, die Ältere entlassen (OECD 2005, S. 127). Zudem gibt es außerhalb der gesetzlichen Bestimmungen in vielen Tarifverträgen besondere Kündigungsschutzregelungen für Ältere. Der hier häufig zu findende Ausschluss der ordentlichen Kündigung mit Erreichen einer bestimmten Altersgrenze setzt jedoch in fast allen Tarifverträgen auch eine bestimmte, längere Betriebszugehörigkeitsdauer voraus (Bispinck/WSI-Tarifarchiv 2005, S. 5). Von Regelungen, die an eine lange Betriebszugehörigkeit anknüpfen, profitieren Ältere zwar öfter als Jüngere; sie haben jedoch auf die Bereitschaft, einen Älteren neu einzustellen, keine Wirkung.
} 
bensalter eines der vier Kriterien, die bei einer betriebsbedingten Kündigung für die Sozialauswahl zu beachten sind. Diese Kriterien sind jedoch nur Leitlinien, die dem Arbeitgeber große Freiheiten bei der Gewichtung lassen. Er kann bei einer uneindeutigen Auswahlsituation selbst entscheiden, welchem der vier Kriterien er den Vorrang gibt. Da zudem über das Kriterium "Alter" Arbeitsmarktchancen und versorgungsrechtliche Absicherung in die Sozialauswahl einfließen sollen, kann auch gerade ein niedriges Lebensalter zu einer besonderen Schutzbedürftigkeit führen. Zudem nimmt die Schutzbedürftigkeit eines Älteren ab, je näher er dem Rentenalter kommt. Eine nennenswerte Erschwernis bei der Kündigung von älteren Arbeitnehmern geht demnach selbst von der Sozialauswahl nicht aus. Die besondere Befristungsregelung für Ältere zielt daher auf die Beseitigung eines nicht vorhandenen Einstellungshindernisses. Sie riskiert den Ausschluss Älterer von dauerhaften Anstellungen unnötigerweise.

\subsection{BEFRISTETE BESCHÄFTIGUNG ALS BRÜCKE?}

Der Gesetzgeber verbindet mit der Neuauflage der Regelung auch die Hoffnung, dass befristete Beschäftigung für die Älteren eine Brücke zu einer dauerhaften Beschäftigung sein wird (BMAS 2006b, S. 13). Als Brücke in eine dauerhafte Beschäftigung sind befristete Beschäftigungen aber nur bedingt tauglich. Eine befristete Beschäftigung erhöht zwar im Vergleich zu einem Verbleib in der Arbeitslosigkeit nachweislich die Chance, drei Jahre später beschäftigt zu sein, um $15 \%$ (Boockmann 2006, S. 17ff.). Eine Befristung birgt aber zugleich ein größeres Risiko, wieder arbeitslos zu werden. Der Vergleich zwischen unbefristeten und befristeten Beschäftigten macht deutlich, dass der Anteil der Arbeitslosen bei den ehemals befristet Beschäftigten um 7,9\% bei den Männern und um $2,9 \%$ bei den Frauen höher war als bei den zuvor unbefristet Beschäftigten (Giesecke/Groß 2006, S. 251). Auch bei einem Vergleich der Beendigungsarten von Arbeitsverhältnissen zeigt sich, dass die Beendigung durch Fristablauf verglichen mit allen anderen Beendigungsarten die größte Gefahr birgt, lange arbeitslos zu bleiben. $91 \%$ derjenigen, die selbst gekündigt hatten, $62 \%$ der vom Arbeitgeber Gekündigten und $71 \%$ derjenigen, die einen Aufhe- bungsvertrag geschlossen hatten, jedoch nur $58 \%$ der zuvor befristet Beschäftigten waren einer Untersuchung zufolge $25 \mathrm{Mo-}$ nate später beschäftigt (Bielinski et al. 2002, S. 445). Befristete Stellen können demnach Arbeitslosen einen leichteren Wiedereinstieg in den Arbeitsmarkt ermöglichen, führen aber nicht zu einer langfristigen Integration.

Die geplante Ausdehnung der allgemeinen zweijährigen Höchstbefristungsdauer auf fünf Jahre für die Älteren verringert zudem - als Nebenwirkung der erhofften größeren Wiedereingliederungschancen Arbeitsloser - die Chance auf einen unbefristeten Vertrag. Ein Arbeitgeber wäre nicht mehr gezwungen, einen Arbeitnehmer, den er weiterbeschäftigen möchte, nach zwei Jahren in eine unbefristete Anstellung zu übernehmen. Auch wenn in einigen Fällen über eine befristete Anstellung der Einstieg in eine dauerhafte Beschäftigung gelingt, ist eine allmähliche Verdrängung von dauerhaften Anstellungen durch befristete der Preis für die verbesserten Aussichten auf eine befristete Anstellung. Aufgrund dieses Effekts muss langfristig die mit der Idee der Brückenfunktion verbundene Hoffnung, die Chancen auf eine Festanstellung zu erhöhen, zwangsläufig enttäuscht werden.

Wenig plausibel sind in diesem Kontext auch jene Argumente, die darauf verweisen, dass durch befristete Beschäftigung die verbleibende Zeit bis zur Rente überbrückt werden kann. Übersehen wird, dass ein über 52-Jähriger im längsten Fall immerhin noch 15 Jahre bis zum Erreichen der zukünftigen Regelaltersgrenze arbeiten muss, was gut ein Drittel seines Erwerbslebens ist. ${ }^{5} \mathrm{Zu}$ bedenken sind darüber hinaus die Konsequenzen eines gelockerten Kündigungsschutzes für die Arbeitsbedingungen im Allgemeinen: Wer im Fall einer tageweisen Befristung überhaupt keinen Kündigungsschutz hat, muss jede Verschlechterung seiner Arbeitsbedingungen akzeptieren. Nachweislich nehmen befristet Beschäftigte seltener als Festangestellte an betrieblicher Weiterbildung teil und müssen ungünstigere Arbeitsbedingungen und verminderte Einkommenschancen hinnehmen (Giesecke/Groß 2006, S. 247, 252 m.w.N).

\section{Reformperspektiven}

Die Reformbilanz der letzten Jahre fällt ernüchternd aus. Leistungen für Ältere wurden nachhaltig eingeschränkt, ohne dass die eingeführten Instrumente die Integrationschancen dieser Personengruppe verbessert hätten. Auch das geplante Festhalten an einem gelockerten Kündigungsschutz für ältere Arbeitnehmer wird deren Arbeitsmarktchancen nicht erhöhen. Vielmehr führt der eingeschlagene Reformweg vermehrt zu prekären Altersübergängen.

Trotz der Erfolglosigkeit der aktuellen Arbeitsmarktpolitik beschränkt sich die Debatte auf den weiteren Ausbau des vorhandenen Instrumentariums. So ist in dem Entwurf für ein Verbesserungsgesetz vorgesehen, die Entgeltsicherung - ein bislang wirkungsloses Instrument - weiter auszubauen. Auch das Institut für Arbeitsmarktund Berufsforschung befürwortet in einem jüngst publizierten Bericht ein Kombilohnmodell für Ältere (Dietz et al. 2006). Ob der Ausbau dieser Regelungen allein zu einer verbesserten Arbeitsmarktintegration älterer Erwerbsloser beitragen kann, ist fraglich. Ein Blick über die Grenzen jedenfalls zeigt, dass Lohnsubventionen oder Nachlässe bei Steuern und Sozialabgaben nur dann Erfolg versprechen, wenn sie Bestandteil einer umfassenden Strategie zur Förderung Älterer sind (Adamy 2006). Der beruflichen Weiterbildung kommt dabei ebenso wie der Ausgestaltung von Erwerbsminderungsrenten eine hohe Bedeutung zu.

Dem Aktivierungsansatz zum Trotz ist auch davon auszugehen, dass ein Teil der Älteren insbesondere aus gesundheitlichen Gründen dem Arbeitsmarkt nicht bis zum Erreichen der Regelaltersgrenze zur Verfügung stehen kann. Gerade in beschäftigungspolitisch erfolgreichen Ländern kommt - trotz weitreichender Einschränkungen der Frühverrentungsmöglichkeiten

\footnotetext{
5 Uneingeschränkt gilt diese Feststellung für die 1962 oder später Geborenen. Sie werden bei Abschluss der schrittweisen Heraufsetzung des Rentenalters 2029 erst mit 67 Jahren in Rente gehen können. Die Altersgrenze von 52 Jahren würden sie in frühestens 6 Jahren erreichen. Bis dahin gilt also, dass die Höchstdauer der noch verbleibenden Arbeitszeit eines 52-Jährigen 13 Jahre beträgt.
} 
- den Ausstiegspfaden über die Erwerbsminderungsrenten eine nach wie vor große Bedeutung zu. So liegt der Anteil aller Erwerbsunfähigen an den Älteren (55 bis 64 Jahre) in Deutschland bei nur $4 \%$. Ein wesentlicher Grund hierfür sind die sehr restriktiven Zugangskriterien in Deutschland. Erwerbsunfähig sind nur diejenigen, die keine drei Stunden am Tag arbeiten können. In den Niederlanden werden gute $14 \%$ aller Älteren durch die Sicherungssysteme bei Erwerbsunfähigkeit geschützt. Dort gelten all jene als erwerbsunfähig, die aus gesundheitlichen Gründen nicht mehr das verdienen können, was gesunde Arbeitnehmer mit vergleichbarer Qualifikation verdienen könnten. Dies hat den Effekt, dass gesundheitlich angeschlagene Personen Leistungen aus dem Sozialsystem bei Erwerbsunfähigkeit erhalten und eben nicht in den Arbeitslosengeldbezug verwiesen werden.
Der Erhalt von Ausstiegsmöglichkeiten für gesundheitlich beeinträchtigte Arbeitnehmer ist folglich unabdingbar. Gleiches gilt für die berufliche Weiterbildung. Sie ist zentrale Voraussetzung für die Beschäftigungsfähigkeit älterer Arbeitnehmer. Zu begrüßen ist zunächst der Ausbau der Weiterbildungsförderung nach $\$ 417$ SGB III, mit dem die Politik ihren Willen bekräftigt, die Förderung älterer Arbeitnehmer voranzutreiben. Arbeitnehmer sollen laut dem geplanten Verbesserungsgesetz bereits mit Vollendung des 45. Lebensjahres (nicht erst ab dem 51. Lebensjahr) und in Betrieben mit bis zu 200 Beschäftigten (bislang 100) durch die Übernahme von Weiterbildungskosten unterstützt werden. Präventiver und integrativer ist jedoch z. B. die Weiterbildungspolitik in Schweden, die über die Erwachsenenbildung Bildungsunterschiede explizit verringern will. Dort erhalten Erwachsene beim Nachholen eines Studien- oder Ausbildungsabschlusses bis maximal zum 55. Lebensjahr eine Unterstützung von $82 \%$ der notwendigen Aufwendungen. Gleichzeitig wird ein „Bildungssparen“ angeboten. Beschäftigte wie Unternehmen zahlen in gleichen Anteilen Geld auf ein Bildungskonto ein, über dessen Guthaben Beschäftigte zu Zwecken der Weiterbildung verfügen können (Adamy 2006, S. 326).

Für die deutsche Politik könnten die genannten Beispiele aus dem Ausland eine Orientierung für die Weiterentwicklung des vorhandenen Systems sein. Anknüpfen könnte man dabei an den Grundgedanken des Arbeitsförderungsrechts von 1969, das Arbeitsmarktpolitik als Ergänzung zur Wachstums- und Strukturpolitik verstanden und ihren präventiven Charakter durch die Förderung von Qualifikationen und Weiterbildung betont hat.

\section{ITERATUR}

Adamy, W. (2006): Warum in Nachbarländern mehr Ältere beschäftigt sind. Was bringt Münteferings „Initiative 50plus“?, in: Soziale Sicherheit 10, S. 322-330

Aust, J./Bothfeld, S./Leiber, S. (2006): Eigenverantwortung - Eine sozialpolitische Illusion?, in: WSI-Mitteilungen 4, S. 186-193

Bundesagentur für Arbeit (BA) (2006a): Eckwerte des Arbeitsmarkts. Der Arbeits- und Ausbildungsmarkt in Deutschland, Dezember und Jahr 2006, Monatsbericht, download unter: http://www.pub.arbeitsamt.de/ hst/services/statistik/000000/html/start/monat/aktuell.pdf Bundesagentur für Arbeit (BA) (2006b): Tabelle III. E.5, Ältere über 50 Jahren in ausgewählten arbeitsmarktpolitischen Maßnahmen, in: Arbeitsmarkt 2005. Amtliche Nachrichten der Bundesagentur für Arbeit, Sonderheft, Nürnberg, S. 189

Bäcker, G. (2006): Rente mit 67: Länger arbeiten oder länger arbeitslos? Probleme einer Heraufsetzung der Altersgrenze, in: Friedrich-Ebert-Stiftung (Hrsg.): Rente mit 67. Steuerungspotenziale in der Renten- und Beschäftigungspolitik, Diskussionspapier, S. 6-23

Bellmann, L./Leber, U. (2005): Betriebliche Einstellung älterer Arbeitnehmer, IAB-Betriebspanel 2004, in: Arbeit und Beruf 6, S. 169-170 Bielinski, H./Hartmann, J./Mauer, A./Seifert, H. (2002): Übergänge zu einem neuen Arbeitsplatz, in: WSI-Mittelungen 8, S. 442-450 Bispinck, R./WSI-Tarifarchiv (2005): Informationen zur Tarifpolitik. Tarifliche Senioritätsregelungen, Düsseldorf

Blos, K./Rudolph, H. (2005): Verlierer, aber auch Gewinner, IAB-Kurzbericht 17 vom 7.10 .
Boockmann, B. (2006): Chancen trotz befristetem Arbeitsvertrag? Arbeitspapier 121 der Hans-Böckler-Stiftung Bosch, G./Schief, S. (2005): Die Beschäftigung Älterer in Europa zwischen Vorruhestand und „Work-Line“ , in: Arbeit 4, S. 275-291 Bothfeld, S. (2005): Aktiv und aktivierend: Grundzüge einer zukunftsfesten Arbeitsmarktpolitik, in: WSI-Mitteilungen 8, S. 419-424 Brussig, M./Knuth, M. (2006): Altersgrenzenpolitik und Arbeitsmarkt Zur Heraufsetzung des gesetzlichen Rentenalters, in: WSI-Mitteilungen 6, S. 307-313

Bundesministerium für Arbeit und Soziales (BMAS) (2006a): Die Wirksamkeit moderner Dienstleistungen am Arbeitsmarkt. Bericht des Bundesministeriums für Arbeit und Soziales zur Wirkung der Umsetzung der Vorschläge der Kommission Moderne Dienstleistungen am Arbeitsmarkt Bundesministerium für Arbeit und Soziales (BMAS) (2006b): Entwurf eines Gesetzes zur Verbesserung der Beschäftigungschancen älterer Menschen, BT-Ds. 16/3793

Deutscher Gewerkschaftsbund (DGB) (2006): Europarechtskonforme Ausgestaltung der so genannten 52er-Regelung, in: Kurzinformation 11, S. $2-4$

Dietz, M./Gartner, H./Koch, S./Walwei, U. (2006): Kombilohn für ältere Arbeitslose. Maßgeschneidert ist besser als von der Stange, IAB Kurzbericht 18 vom 20.10.

Engstler, H./Brussig, M. (2006): Arbeitslosigkeit am Ende des Erwerbslebens, in: Informationsdienst Altersfragen 6, S. 2-6 
Eichhorst, W./ Sproß, C. (2005): Die Weichen führen noch nicht in die gewünschte Richtung, IAB-Kurzbericht 16 vom 5.10.

Europäischer Gerichtshof (EuGH) (2005): Mangold-Urteil des EuGH vom 22.11.2005, Az. C 144/04, in: Neue Zeitschrift für Arbeitsrecht, S. 13451348

Gangl, M./Schmidt, G. (2002): Anreizwirkungen der Arbeitslosenversicherung, in: Wirtschaftsdienst 8, S. 451-455

Giesecke, J./Groß, M. (2006): Befristete Beschäftigung, in: WSI-Mitteilungen 5, S. 247-254

Knuth, M./Büttner, R./ Brussig, M. (2006): Ein längeres Arbeitsleben für alle? Aktuelle renten- und arbeitsmarktpolitische Entwicklungen im Lichte der Ergebnisse des "Altersübergangs-Reports", download unter: http://www.iatge.de/auem-report/2006/2006-01/auem2006-01.pdf Kraatz, S./Rhein, T./Sproß, C. (2006): Bei der Beschäftigung Älterer liegen andere Länder vorn, IAB-Kurzbericht 5 vom 3.4.

Lotter, W. (2006): Ohne Zweifel, in: brandeins 11, S. 58-70

OECD (2004): Employment Outlook, Paris

OECD (2005): Alterung und Beschäftigungspolitik. Deutschland, Paris

Preis, U. (2006): Verbot der Altersdiskriminierung als Gemeinschafts-

grundrecht. Der Fall "Mangold“ und die Folgen, in: Neue Zeitschrift für

Arbeitsrecht 8, S. 401-456
Promberger M./Wübbecke C. (2006): Pro und Contra Rente mit 67, IAB Kurzbericht 8 vom 16.5.

Sachverständigenrat (SVR) (2006): Widerstreitende Interessen - Ungenutzte Chancen, Jahresgutachten 2006/2007, Berlin

Seifert, H. (2006): Was hat die Flexibilisierung des Arbeitsmarktes gebracht? in: WSI-Mitteilungen 11, S. 601-607

Sesselmeier, W. (2004): Deregulierung und Reregulierung der Arbeitsmärkte im Lichte der Insider-Outsider-Theorie, in: WSI-Mitteilungen 3, S. 125-131

Thüsing, G. (2005): Europäischer Gleichbehandlungsgrundsatz als Bindung des Arbeitgebers?, in: Zeitschrift für Wirtschaftsrecht und Insolvenzpraxis, S. 2149-2151

Wiedemann, H./Thüsing, G. (2002): Der Schutz älterer Arbeitnehmer und die Umsetzung der Richtlinie 2000/78/EG, in: Neue Zeitschrift für Arbeitsrecht, S. 1234-1242

WSI (2001): Befragung zur Beendigung von Arbeitsverhältnissen, teilweise (Tabelle 1 nicht) veröffentlicht in: Pfarr, H./Ullmann, K./Bradtke, M./ Schneider, J./Kimmich, M./Bothfeld, S. (2005): Der Kündigungsschutz zwischen Wahrnehmung und Wirklichkeit, München und Mering

\section{Die Steuerpolitik der großen Koalition auf dem Prüfstand}

Workshop des WSI-/IMK-Arbeitskreises Steuerpolitik WSI und IMK in der Hans-Böckler-Stiftung

8. Mai 2007 in Berlin

$\mathrm{Zu}$ folgenden Themen referieren und diskutieren $\mathrm{u}$.a.:

Die Unternehmenssteuerreform 2008: Eine kritische Bewertung

Prof. Dr. Lorenz Jarass, FH Wiesbaden;

Unternehmenssteuern in Europa: Aktuelle Trends

Dr. Margit Schratzenstaller, WiFO Wien;

Kritisches zur Erbschaftsteuerreform

Prof. Dr. Joachim Wieland, Universität Frankfurt;

Die geplante Abgeltungsteuer

Dr. Stefan Bach, DIW Berlin;

Möglichkeiten der Verbesserung des Steuervollzugs

Dieter Ondracek, Vorsitzender der Deutschen Steuergewerkschaft;

Grußwort: Die Steuerpolitik der großen Koalition aus Sicht der IG Metall

Wolfgang Rode, IG Metall, Mitglied des geschäftsführenden Bundesvorstands

Anmeldung/Information: Heike Stempel, Hans-Böckler-Stiftung, e-mail: heike-stempel@boeckler.de 